\title{
Vibration tests and metamodelling for composite material characterisation
}

\author{
S. Syngellakis ${ }^{1} \&$ R. Setiawan ${ }^{2}$ \\ ${ }^{1}$ Wessex Institute of Technology, UK \\ ${ }^{2}$ Institut Teknologi Bandug, Indonesia
}

\begin{abstract}
The mechanical characterisation of laminates is achieved through a methodology based on vibration test measurements. The relation between effective mechanical properties and vibrational characteristics is initially generated by multiple finite element simulations and then approximated through the development of a surrogate model or meta-model. The inverse problem of finding the properties from given experimental data is solved through an iterative optimisation scheme within which the frequencies for a particular set of material parameters are predicted by the meta-model. This results in a versatile and time-saving characterisation process, which can be applied to a large number of specimens with a complicated geometry.
\end{abstract}

Keywords: composites, characterisation, vibration, experiments, metamodelling.

\section{Introduction}

Advanced composites have become realistic alternatives to conventional materials for the design of safe and durable structures since they can meet the requirements for high strength- or stiffness-to-weight ratios and other structural integrity objectives. A reliable dynamic performance is another important requirement on composites in many applications. For an accurate analytical assessment of composite behaviour under loads, their mechanical properties need to be known with sufficient confidence and precision. Such properties can be determined by static tests, according to established procedures [1], which involve several specimens cut from the same material. 
Early work on composite characterisation through dynamic tests was based on a relatively simple experimental technique according to which the composite properties are determined iteratively from experimentally obtained modal parameters; this approach was initially developed for and applied to orthotropic plates $[2,3]$. The mode shapes associated with the measured natural frequencies may be included in the characterisation process. A direct approach $[4,5]$ required a precise mode shape measurement but another [6] only qualitative examination of the mode shapes associated with the measured natural frequencies. Various optimisation algorithms can be used for the solution of the inverse problem of determining properties from experimental vibration output [7-9].

The development of components for various applications demands composites with increasingly complicated geometry, such as laminates with stiffening, sandwich structures, plates with variable thickness. Moreover, mass production of composite components requires fast, inexpensive characterisation, preferably carried out in the laboratory, subsequent to the dynamic tests [10]. Existing strategies are computationally expensive when applied to samples with complex geometries.

This paper presents an alternative strategy for the solution to this problem by making use of meta-modelling to approximate the relation between natural frequencies and material properties. Such an approximate model is versatile with respect to geometric and material parameter selection and efficiently applicable to the required solution of an inverse problem, leading to fast, in-situ composite characterisation.

\section{Methodology}

The material property parameters and their ranges are initially selected. This process is either guided by or guides the preparation of the specimens. Dynamic tests are performed resulting in sets of measured natural frequencies. An FE program relating material to vibration parameters is built and validated by comparing its predictions to the experimental data. A number of material property sets are generated by a scheme that guarantees a good spread of data over their range. Multiple FE analyses are then carried out with these property sets as inputs and natural frequencies sets as outputs. Thus, data sets comprising both independent and dependent variables are generated and subsequently used to train meta-models. The latter are simple approximate surrogates of the more expensive and time consuming FE analysis. Finally, an optimisation algorithm is used for the solution of the inverse problem, namely the determination of material properties from available vibration data.

The adopted material parameter set consisted of the in-plane elastic constants $E_{x}, E_{y}, G_{x y}$ and $v_{x y}$ of laminated, orthotropic plate specimens. A large number of such sets were generated according to a random sampling method, subject to the constraints that the material properties lie within specified ranges and the resulting stiffness matrix of the material is positive-definitive. For the latter condition to be satisfied, the four in-plane constants obtained from the sampling were complemented by assumed values for the through-thickness properties. 
Each material set was input to the FE vibration analysis yielding a set of frequencies $f_{i}$. The rectangular plate specimens were modelled by a $12 \times 12$ mesh of quadrilateral, 8-node shell elements. The adopted element type is based on a first order shear deformation theory (FSDT), which is expected to give accurate natural frequency predictions for plates with moderate thickness to width ratio. It requires input of effective laminate properties, including the four in-plane elastic constants.

The independent variable set actually employed in the characterisation process comprised the values of the frequency parameter, defined by

$$
\bar{f}_{i}=f_{i} h \sqrt{\rho}
$$

where $h$ is the thickness of the specimen and $\rho$ the average density of the material.

The developed meta-models provided an approximation to the relation between material and vibration parameters generated by FE modelling. They were based on the Gaussian radial basis function (RBF), which takes the form,

$$
y(\mathbf{x})=\sum_{j=1}^{m} w_{j} \exp \left(-\frac{\left(\mathbf{x}-\mathbf{c}_{j}\right)^{2}}{r^{2}}\right)
$$

where $y(\mathbf{x})$ is the approximated function, $m$ is the number of basis functions; $w_{j}$ are the weights; $\mathbf{x}$ is the array of input variables; $\mathbf{c}_{j}$ the array of the centres, and $r$ is the width parameter, here taken as constant for all centres.

Eqn (2) was evaluated for each input-output pair of the training data with the centres being defined as coincident with the training data. The weights, $w_{j}$, can be found by minimising a cost function defined by the sum of squared errors (SSE) of model responses (here the natural frequencies) between the training data and those predicted by the meta-model. There are various techniques for improving the accuracy and efficiency of meta-models, including optimising the choice of training data to be used [11].

For the characterisation, the meta-models need to be based on specific specimen geometry, leaving only the material properties as the variables to be identified. For plate specimens, the length-to-thickness and aspect ratios can be considered constant, so that the same meta-model can be applied to specimens having such ratios with the same values. The thickness and density are incorporated in the meta-model output, that is, the frequency parameters as defined by eqn (1).

An accuracy test method, based on the $R^{2}$ accuracy measure, may be employed. This measure is given by

$$
R^{2}=1-\frac{\sum_{i=1}^{N}\left(y_{i}-\hat{y}_{i}\right)^{2}}{\sum_{i=1}^{N}\left(y_{i}-\bar{y}_{i}\right)^{2}}
$$

where $y_{i}, \hat{y}_{i}, \bar{y}$ are the RBF predictions, true and the mean of the true values, respectively, observed at $p$ validation points. 
The optimisation problem involved the minimisation of the error function

$$
\phi=\sum_{i=1}^{N} \frac{\left(\bar{f}_{i}-\tilde{f}_{i}\right)^{2}}{\tilde{f}_{i}^{2}}
$$

where $N$ is the number of frequencies being considered and $\tilde{f}_{i}$ the experimentally obtained frequency parameters. The search for the optimum solution to the characterisation problem was subject to the same constraints on material properties as those imposed on the selection of training data. These constraints were implemented within the optimisation algorithm using the external penalty function method, which tackles solutions that violate them by adding a penalty and forcing the solution in the direction of the feasible region. By introducing these external penalty terms and thus formulating a revised objective function, an unconstrained optimisation algorithm can be used to solve a constrained optimisation problem. An algorithm called BFGS (Broyden, Fletcher, Goldfarb, Shanno) [12], was used; this algorithm falls into the non-linear unconstrained optimisation category, based on the steepest descent method with global convergence capability.

\section{Experiments}

The vibration experiments were designed to simulate free edge conditions for rectangular plate specimens. Such conditions lead to accurate measurement of natural frequencies; for this reason, they have been widely adopted in the past despite the absence of an explicit solution to the corresponding theoretical problem.

Setting up a versatile experimental arrangement yielding accurate estimates of the lower natural frequencies required the design of supports approximating freeedge conditions and the selection of excitation apparatus, natural frequency measurement methods as well as appropriate sensing devices. These features should have a negligible constraint or inertia effect on the measured frequencies; if this is not possible, the effect should be compensated or accounted for in the modelling. The information on the mode shapes associated with the measured natural frequencies also contributes to the accuracy of the characterisation. Therefore, an experimental arrangement was achieved yielding this information.

One possible, simple method of replicating ideal free-edge conditions is the soft-pad support [3]. This reduces the dominance of the rigid body vibration mode but the imposed lateral constraint can be considerable even if a spikedshape sponge is used to minimise contact between support and specimen. For this reason, the more widely used method of specimen suspension $[2,7,13,14]$, as shown in fig. 2, was also adopted here.

The experiments were carried out using a mechanical shaker, which imposed continuous pseudo-random excitation on the specimen and, as sensor, a laser vibrometer, which allows non-contacting sequential measurement at several points thus reducing the possibility of overlooking any vibration mode. The natural frequencies for each specimen were extracted from the average of all measurements taken from the entire specimen domain, not just from a single 
point. From the response spectra and phases of all points of measurement, the vibration mode shape at each frequency can be systematically deduced and viewed on the computer screen through post-processing analysis.

The laser vibrometer equipment consisted of a laser-emitter/digital-camera unit, signal processor/controller unit and a PC as a display, post processing and storage device as schematically shown in fig. 3. The plate was suspended from wires and the excitation was imposed at approximately the centre point of the lower left quadrant of the specimen. The shaker was screwed to the specimen to provide a sufficiently fixed attachment.

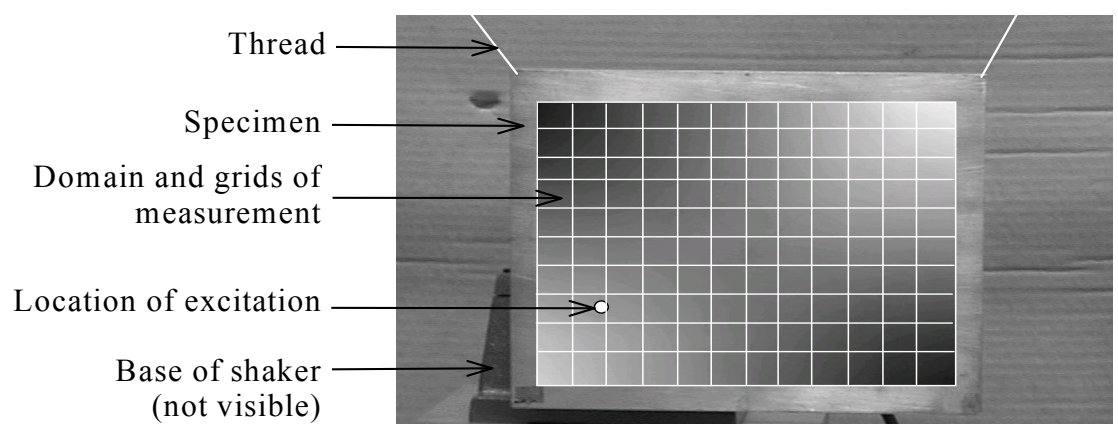

Figure 1: Dynamic response of suspended specimen as shown in the equipment display.

First, a pseudo-random excitation signal was defined within a certain frequency range. The image of the specimen was captured through the digital camera and displayed on the PC screen. Then, a piece of software within the PC unit (5) defined a mesh over the valid measurement area on the specimen as shown in fig. 2. The commands were then realised by the controller (4), to generate the excitation signal that was sent to the signal amplifier (6) and finally the shaker (2). The commands related to data acquisition were sent to the laser/camera unit (3).

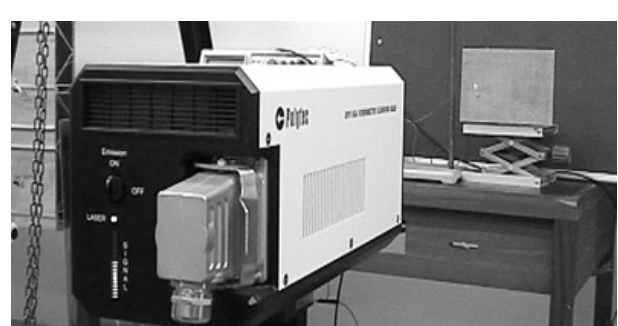

(a)

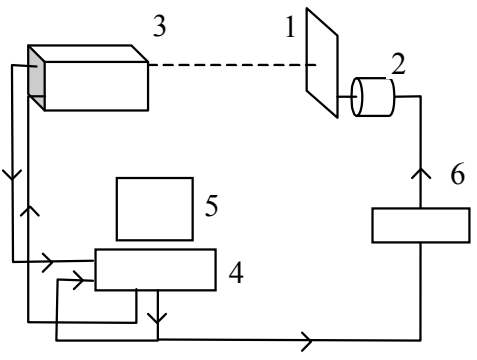

(b)

Figure 2: (a) Laser vibrometer and (b) schematic diagram of the corresponding set up: specimen (1), mechanical shaker (2), laser/camera unit (3), controller (4), PC unit (5), signal amplifier (6). 
The effectiveness of the test arrangements to generate accurate frequency measurement was tested by using initially a $155 \times 200 \times 1.15 \mathrm{~mm}^{3}$ aluminium plate as the specimen. The modulus of elasticity, shear modulus and Poisson's ratio were taken equal to $72.4 \mathrm{GPa}, 28 \mathrm{GPa}$ and 0.33 , respectively. The natural frequencies were extracted from the average spectrum shown in fig. 4. As can be seen in this figure, the averaging process results in a high signal-to-noise ratio.

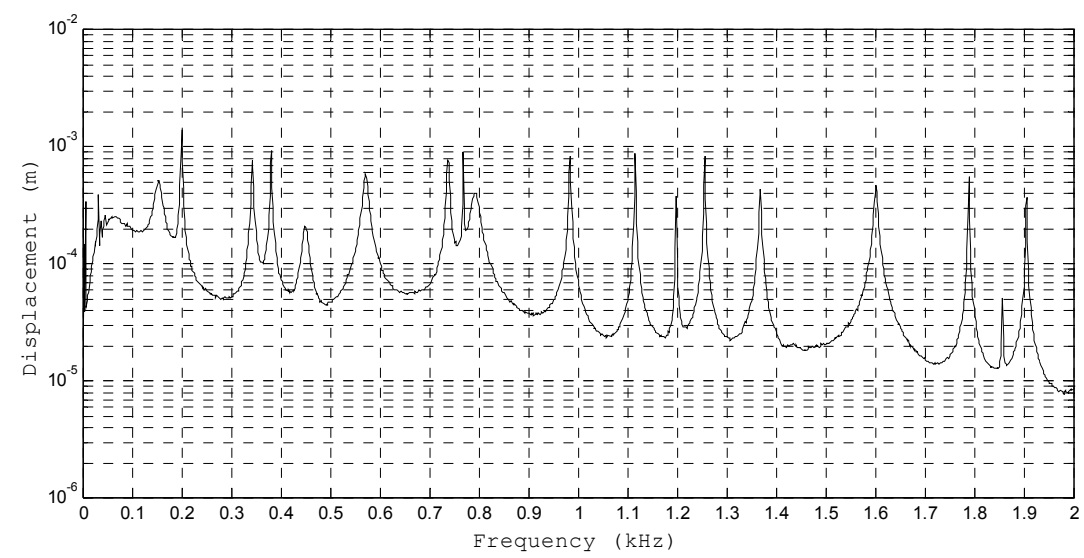

Figure 3: Average frequency response function for the aluminium specimen.

The frequency peaks are clearly identified and accepted as natural frequencies. Frequency peaks below $100 \mathrm{~Hz}$ were probably associated with either rigid body vibration modes or electromagnetic interference. The first significant frequency peak is at approximately $150 \mathrm{~Hz}$. The mode shapes at that frequency and below indicated that the one at $150 \mathrm{~Hz}$ corresponded to the twisting mode, thus interpreted as the fundamental frequency; frequencies below $100 \mathrm{~Hz}$ corresponded to rigid body vibration modes.

The main issue with a mechanical shaker, attached to the specimen by a connector, is the possibility of the excitation having a significant effect on the plate vibration characteristics. For this reason, the experimental results were compared with predictions from two FE models, namely one including the modelling of the shaker-connector arrangement (FE 1) and another without it (FE 2). The frequencies extracted from fig. 4 are listed in table 1 together with the corresponding analytical results with their percentage differences given in square brackets. The mode shapes are shown in grey shade, the experimental ones from white to dark and the analytical ones from dark to white and to dark.

The results of table 1 clearly indicate that FE 1 , that is, the FE model that includes the shaker-connector arrangement generally leads to predictions in better agreement with the experimental natural frequencies and associated vibration modes. With this model, the biggest discrepancy between theory and experiment occurs in the sixth mode $(9.34 \%)$ whereas, neglecting the connector effect, the most significant prediction shift compared with the respective experimental result occurs in the ninth frequency, where the difference is $15.63 \%$. 
Table 1: Experimental and analytical natural frequencies and associated mode shapes of a suspended aluminium plate.

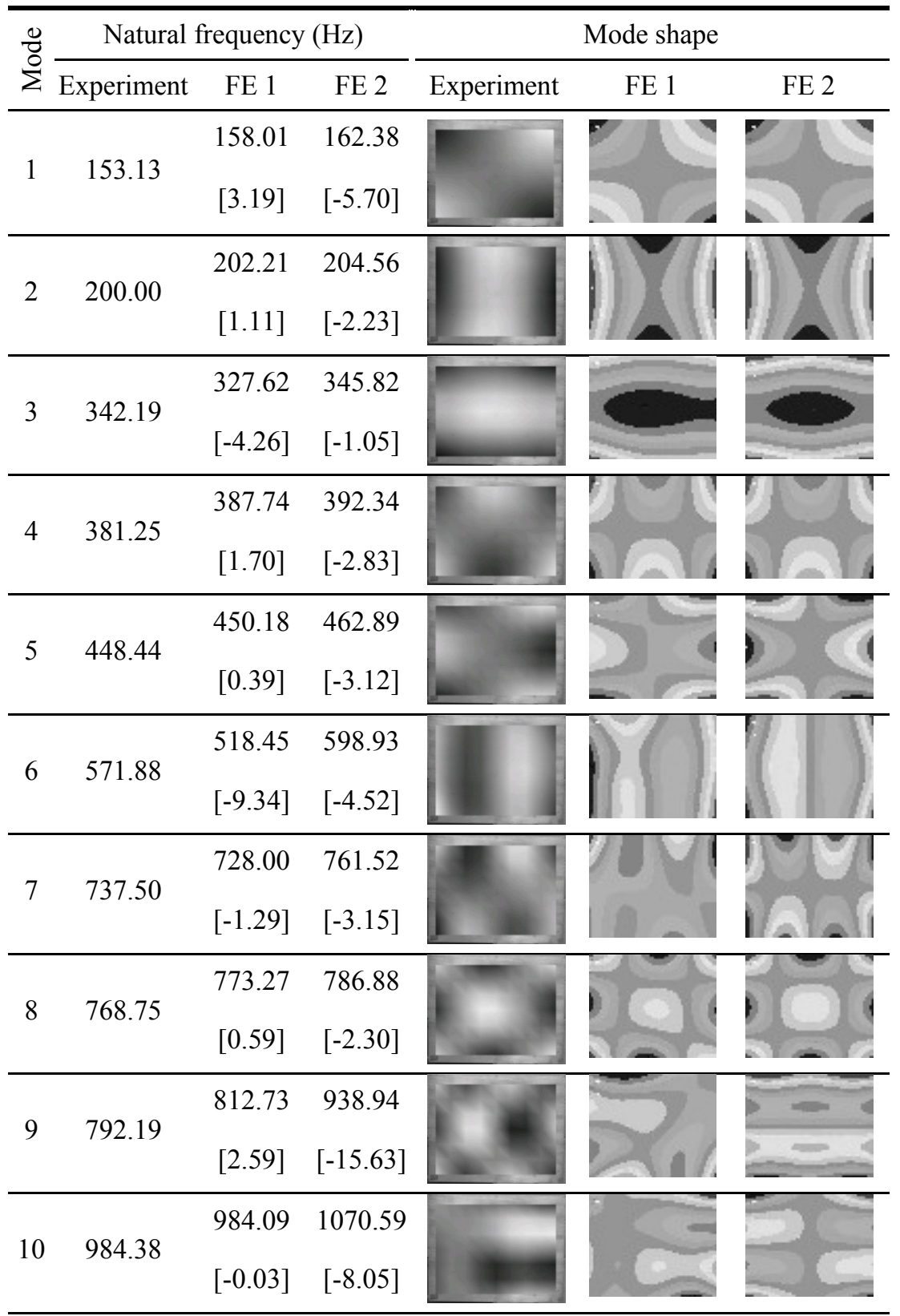




\section{Characterisation results}

The proposed characterisation strategy was applied, as a test case, to a generic glass-fibre/epoxy-resin based composite plate; this was consistent with the characteristics of the available specimens. The adopted ranges of material properties were

$$
\begin{gathered}
2 \leq E_{x} \leq 45 \quad(\mathrm{GPa}) \\
2 \leq E_{y} \leq 30 \quad(\mathrm{GPa}) \\
1 \leq G_{x y} \leq 20 \quad(\mathrm{GPa}) \\
0.05 \leq v_{x y} \leq 0.8
\end{gathered}
$$

The bounds in the ranges (5) were specified according to available design data for this particular type of material so that they were as narrow as possible; this is expected to improve the accuracy of metamodelling.

The specimens were laminates with various ply arrangements and thicknesses; their properties were within the bounds (5). The characteristics of the four specimens tested are presented in table 2. The length-to-thickness ratio of the specimens was intended equal to either 55 or 110 while the aspect ratio was intended equal to 1.4. For this reason, two meta-models were built, based on training data generated using these values.

Table 2: $\quad$ Details of the four test laminate specimens.

\begin{tabular}{ccl}
\hline Laminate & $V_{f}$ & \multicolumn{1}{c}{ Description } \\
\hline A & 0.46 & 3-layer 600 g/m $/ \mathrm{m}^{2}$ woven roving (WR) \\
B & 0.19 & 3-layer 300 g/m $/ \mathrm{m}^{2}$ chopped strand mat (CSM) \\
C & 0.33 & 6-layer 225 g/m $/ \mathrm{m}^{2} \mathrm{CSM} / 600 \mathrm{~g} / \mathrm{m}^{2} \mathrm{WR}( \pm 45)$ \\
$\mathrm{D}$ & 0.18 & 3-layer 600 g/m $/ \mathrm{m}^{2}$ chopped strand mat (CSM) \\
\hline
\end{tabular}

Measurement Data:

\begin{tabular}{cccccc} 
Laminate & $h(\mathrm{~mm})$ & $a(\mathrm{~mm})$ & $b(\mathrm{~mm})$ & Mass $(\mathrm{g})$ & Density $\left(\mathrm{kg} / \mathrm{m}^{3}\right)$ \\
\hline A & 1.68 & 204 & 145.5 & 82.5 & 1653.0 \\
B & 1.85 & 195 & 141 & 70.5 & 1387.1 \\
C & 2.92 & 161.5 & 117 & 85.3 & 1542.1 \\
D & 3.89 & 206.3 & 149.3 & 166.1 & 1387.0 \\
\hline
\end{tabular}

As with the aluminium specimen, the natural frequencies of each composite specimen were extracted from the average of all measurements taken over the entire specimen domain, not just from a single point. The natural frequencies extracted from these average spectra are listed in table 3 for up to twelve modes, excluding the low frequencies at around $50 \mathrm{~Hz}$. The frequency parameters were calculated from eqn (1) using the measured thickness and density values listed in table 2 . 
Table 3: $\quad$ Measured natural frequencies and respective frequency parameters.

\begin{tabular}{|c|c|c|c|c|c|c|c|c|}
\hline \multirow[b]{2}{*}{${ }^{0}$} & \multicolumn{2}{|c|}{ Laminate A } & \multicolumn{2}{|c|}{ Laminate B } & \multicolumn{2}{|c|}{ Laminate C } & \multicolumn{2}{|c|}{ Laminate D } \\
\hline & $f(\mathrm{~Hz})$ & $\bar{f}$ & $f(\mathrm{~Hz})$ & $\bar{f}$ & $f(\mathrm{~Hz})$ & $\bar{f}$ & $f(\mathrm{~Hz})$ & $\bar{f}$ \\
\hline 1 & 92.50 & 6.324 & 107.50 & 7.401 & 267.50 & 30.700 & 200.00 & 28.949 \\
\hline 2 & 137.50 & 9.400 & 131.25 & 9.036 & 298.75 & 34.286 & 239.06 & 34.603 \\
\hline 3 & 233.75 & 15.980 & 241.25 & 16.609 & $328.75^{b}$ & 37.729 & 478.13 & 69.206 \\
\hline 4 & 262.50 & 17.946 & 260.00 & 17.900 & $352.50^{\mathrm{b}}$ & 40.455 & 504.69 & 73.051 \\
\hline 5 & 315.00 & 21.535 & 326.25 & 22.461 & 603.75 & 69.290 & 604.69 & 87.525 \\
\hline 6 & 372.50 & 25.466 & 368.75 & 25.387 & 673.75 & 77.323 & 720.31 & 104.261 \\
\hline 7 & 470.00 & 32.131 & 490.00 & 33.734 & 771.25 & 88.513 & 918.75 & 132.984 \\
\hline 8 & 492.50 & 33.669 & 497.50 & 34.251 & 926.25 & 106.301 & 1006.25 & 145.649 \\
\hline 9 & 583.75 & 39.908 & 545.00 & 37.521 & 1227.50 & 140.874 & 1143.75 & 165.551 \\
\hline 10 & 743.75 & 50.846 & 716.25 & 49.311 & 1326.25 & 152.208 & 1354.69 & 196.083 \\
\hline 11 & 756.25 & 51.700 & 780.00 & 53.700 & 1422.50 & 163.254 & 1407.81 & 203.772 \\
\hline 12 & 797.50 & 54.520 & 836.25 & 57.572 & 1673.75 & 192.089 & 1568.75 & 227.067 \\
\hline
\end{tabular}

${ }^{5}$ Ambiguous results.

The experiments were not properly prepared to provide the output required for the application of the characterisation scheme accounting for mode shape information according to the modal assurance criteria (MAC) approach [11]. Thus, the characterisation of the tested laminates was carried out without rigorous consideration of experimentally obtained mode shapes.

The pairs of laminates $(A, B)$ and $(C, D)$ were characterised using the metamodels built with $a / h=110$ and $a / h=55$, respectively. Since however the $a / h$ values for these specimens were not exactly equal to either 110 or 55, correction factors were introduced to compensate for such discrepancies. For this purpose, the variation of $a / h$ with the frequency parameter was obtained using a Ritz vibration analysis based on classical laminate plate theory (CLPT); this meant that higher mode natural frequencies could have been over-estimated. The value of aspect ratio, $a / b$, of the tested specimens was very close to 1.4 , that is, the value adopted in the metamodels, therefore the effect of its variation on the characterisation results was ignored. It is worth noting that a metamodel could have been built for each specimen adopting the exact values of the corresponding $a / h$ and $a / b$ ratios.

The optimum data for metamodels for both cases of $a / h$ ratio can be found in table 4 where $m$ is the number of optimum training data chosen from the 721 admissible training data, $\sigma_{\mathrm{CP}}^{2}$ is the error variance of the confirmation points prediction, $\bar{f}_{C P}^{\min }$ and $\bar{f}_{C P}^{\max }$ represent, respectively, the minimum and maximum values of the predicted frequency parameter from, $e_{m}$ is the maximum error across the confirmation points, and $R^{2}$ is an accuracy measure defined by eqn (3). 
Table 4: $\quad$ Number of optimum training data for metamodelling and selected measures of accuracy, for each mode.

\begin{tabular}{lccccccccc}
\hline \multicolumn{7}{l}{ Optimum metamodelling information for case I $(a / h=110)$} \\
\hline $\begin{array}{l}\text { Mode } \\
\text { no. }\end{array}$ & 1 & 2 & 3 & 4 & 5 & 6 & 7 & 8 & 9 \\
\hline$m$ & 173 & 179 & 176 & 203 & 183 & 208 & 101 & 199 & 140 \\
$\sigma_{\mathrm{CP}}^{2}$ & 0.0195 & 0.0311 & 0.1069 & 0.1511 & 0.1438 & 0.3903 & 1.308 & 0.7499 & 1.3948 \\
$\bar{f}_{C P}^{\min }$ & 3.9273 & 5.6105 & 9.0927 & 12.844 & 14.222 & 17.04 & 21.951 & 24.559 & 30.134 \\
$\bar{f}_{C P}^{\max }$ & 16.642 & 17.85 & 35.943 & 38.509 & 43.336 & 64.16 & 71.447 & 81.731 & 98.545 \\
$e_{m}(\%)$ & 6.94 & 10.66 & 7.36 & 9.17 & 13.92 & 10.10 & 9.56 & 8.69 & 18.84 \\
$R^{2}$ & 0.9979 & 0.9949 & 0.9962 & 0.9949 & 0.9953 & 0.9936 & 0.9864 & 0.9931 & 0.991 \\
\hline Optimum metamodelling information for case $\mathrm{II}(a / h=55)$ & & \\
\hline$m$ & 192 & 207 & 200 & 220 & 158 & 115 & 168 & 175 & 173 \\
$\sigma_{\mathrm{CP}}^{2}$ & 0.2957 & 0.4618 & 1.5787 & 2.6703 & 2.8020 & 11.455 & 14.770 & 12.886 & 22.368 \\
$\bar{f}_{C P}^{\min }$ & 14.961 & 15.198 & 29.798 & 33.593 & 41.627 & 42.503 & 61.069 & 68.462 & 81.991 \\
$\bar{f}_{C P}^{\max }$ & 67.425 & 71.975 & 148.98 & 157.86 & 174.39 & 255.01 & 294.4 & 324.83 & 388.3 \\
$e_{m}(\%)$ & 7.08 & 8.81 & 9.30 & 10.33 & 13.17 & 14.62 & 11.74 & 9.01 & 18.18 \\
$R^{2}$ & 0.998 & 0.9955 & 0.9966 & 0.9945 & 0.9943 & 0.9886 & 0.9905 & 0.9927 & 0.9911 \\
\hline
\end{tabular}

Table 5 shows the characterisation results for the four tested specimens. Each result is the best among those obtained from the five initial guesses. Since there was some uncertainty concerning some frequency measurements, combinations of the most probable frequency parameters among those listed in table 3 were considered in the optimisation process. In the case of laminate $\mathrm{C}$, for instance, the analysis was performed with and without the ambiguous results included in table 3. In such cases, the selection of the most plausible solution can be based on knowledge of the specimen's architecture and composition. The values for $E_{x}$ and $E_{y}$ obtained from static bending tests are included in table 5 for comparison; they should not however be considered as benchmarks for selecting the true solution. The characterisation result meets the expectations arising from the consideration of the tested laminate compositions and architecture.

Table 5: Characterisation results (static test values in brackets).

\begin{tabular}{lllllll}
\hline & \multicolumn{2}{l}{$E_{x}(\mathrm{GPa})$} & \multicolumn{2}{l}{$E_{y}(\mathrm{GPa})$} & $G_{x v}(\mathrm{GPa})$ & $v_{x v}$ \\
\hline Laminate A & 17.67 & $(14.79)$ & 12.96 & $(14.71)$ & 3.98 & 0.05 \\
\hline Laminate B & 8.19 & $(7.08)$ & 6.01 & $(6.43)$ & 4.57 & 0.09 \\
\hline \multirow{2}{*}{ Laminate C } & 3.51 & \multirow{2}{*}{$(7.27)$} & 2.37 & $(6.87)$ & 6.91 & 0.26 \\
& $\underline{8.99}$ & & $\underline{10.77}$ & & $\underline{5.66}$ & $\underline{0.15}$ \\
\hline Laminate D & 6.39 & $(7.46)$ & 9.77 & $(7.66)$ & 4.80 & 0.15 \\
\hline
\end{tabular}

${ }^{a}$ The underlined predictions for laminate $\mathrm{C}$ were obtained with the two ambiguous measurements of 328.75 and $352.50 \mathrm{~Hz}$ excluded. 


\section{Conclusions}

Each of the various experimental and analytical procedures constituting the proposed characterisation scheme may contribute errors to the final outcome. The mass of the moving part of the mechanical shaker and the stiffness of the connector affect the vibration characteristics of the specimen and need to be taken into account in the development of a reliable metamodel. Modelling the essential features of the shaker-connector arrangement for the case of aluminium specimen improved the agreement between experimental and analytical results. Thus, this modelling can be incorporated in the FE analysis for the training data generation. As an alternative, non-contact excitation, for instance, through a magnetic shaker or a speaker generating acoustic waves, would obviate the need for complex modelling and lead to experimental output very close to that predicted for vibrating specimens with free edges.

The use of a laser vibrometer makes possible the application of a scheme that uses experimental mode shape information; such a scheme has been successfully tested with simulated experimental data [11] and should be applied with vibration output from real tests. With regard to the signal processing, a higher frequency resolution would enhance frequency peaks and thus remove ambiguities from natural frequency readings from the frequency response functions. Specimen dimensions and density should be accurately measured and consistent with the respective values used in the metamodel.

The natural frequencies of plates with all-edges free have low sensitivity to Poisson's ratio variation [8]; this inherently leads to a poor result for this material parameter. Such a problem could be addressed by adopting a multi-step characterisation strategy, based on the assumption that natural frequencies have different sensitivity to material properties variation under different support conditions. An early attempt was made at experimental replication of simplesupport boundary conditions for the dynamically tested specimens. However, it was not possible to achieve an experimental set-up yielding reliable frequency output corresponding to simple-support conditions. The multi-step strategy could still be feasible if a more sophisticated experimental apparatus replicating various combinations of support conditions could be devised. For the characterisation of through-thickness mechanical properties, the plate specimen should be sufficiently thick, and the dynamic analysis should take into account the thickness effects accurately.

\section{References}

[1] ASTM, ASTM Standards and Literature References for Composite Materials, ASTM: 1990.

[2] De Wilde, W.P. and Sol, H., Anisotropic material identification using measured resonant frequencies of rectangular composite plates. Composite Structures 4, ed. I.H. Marshal, Paisley College of Technology: Scotland, pp. 317-324, 1987. 
[3] Deobald, L.R. and Gibson, R.F., Determination of elastic constants of orthotropic plates by a modal analysis/Rayleigh-Ritz technique. Journal of Sound and Vibration, 124(2), pp. 269-283, 1988.

[4] Grediac, M. and Paris, P.A., Direct identification of elastic constants of anisotropic plates by modal analysis: Theoretical and numerical aspects. Journal of Sound and Vibration, 195(3), pp. 401-415, 1996.

[5] Grediac, M., Fournier, N., Paris, P.A. and Surrel, Y., Direct identification of elastic constants of anisotropic plates by modal analysis: Experimental results. Journal of Sound and Vibration, 210(5), pp. 643-659, 1998.

[6] Gaul, L., Willner, K. and Hurlebaus, S., Determination of material properties of plates from modal ESPI measurements. Proceedings of the 17th International Modal Analysis Conference, Society for Experimental Mechanics: Bethel, pp. 1756-1752, 1999.

[7] Pedersen, P. and Frederiksen, P.S., Identification of orthotropic material moduli by a combined experimental/numerical method. Measurement, 10(3), pp. 113-118, 1992.

[8] Frederiksen, P.S., Numerical studies for the identification of orthotropic elastic constants of thick plates. European Journal of Mechanics A-Solids, 16(1), pp. 117-140, 1997.

[9] Sol, H., Hua, H., De Visscher, J., Vantomme, J. and De Wilde, W.P., A mixed numerical/experimental technique for the non-destructive identification of stiffness properties of fibre reinforced composite materials. NDT and E International, 30(2), pp. 85-91, 1997.

[10] Gibson, R.F., Modal vibration response measurements for characterization of composite materials and structures. Composites Sciences and Technology, 60, pp. 2769 - 2780, 2000.

[11] Setiawan, R., Syngellakis, S. and Hill, M., A metamodeling approach to mechanical characterization of anisotropic plates. Journal of Composite Materials, 43(21), pp. 2333-2349, 2009.

[12] Kelley, C.T., Iterative Methods for Optimization, SIAM: Philadelphia, 1999.

[13] Araújo, A.L., Mota Soares, C.M. and Moreira de Freitas, M.M., Characterization of material parameters of composite plate specimens using optimization and experimental vibrational data. Composites: Part B, 27B, pp. 185-191, 1996.

[14] De Visscher, J., Sol, H., De Wilde, W.P. and Vantomme, J., Identification of damping properties of orthotropic composite materials using a mixed numerical experimental method. Applied Composite Materials, 4, pp. 1333, 1997. 